\title{
A Streptomyces coelicolor host for the heterologous expression of Type III polyketide synthase genes
}

\author{
Anyarat Thanapipatsiri 1,2, Jan Claesen ${ }^{2,4}$, Juan-Pablo Gomez-Escribano², Mervyn Bibb² \\ and Arinthip Thamchaipenet ${ }^{1,3^{*}}$
}

\begin{abstract}
Background: Recent advances in genome sequencing, combined with bioinformatic analysis, has led to the identification of numerous novel natural product gene clusters, particularly in actinomycetes of terrestrial and marine origin. Many of these gene clusters encode uncharacterised Type III polyketide synthases. To facilitate the study of these genes and their potentially novel products, we set out to construct an actinomycete expression host specifically designed for the heterologous expression of Type III PKS genes and their gene clusters.

Results: A derivative of Streptomyces coelicolor A3(2) designed for the expression of Type III polyketide synthase (PKS) genes was constructed from the previously engineered expression strain S. coelicolor M1152 [ $\Delta$ act $\Delta$ red $\Delta c p k \Delta c d a$ rpoB(C1298T)] by removal of all three of the endogenous Type III PKS genes (gcs, srsA, rppA) by PCR targeting. The resulting septuple deletion mutant, M1317, proved to be an effective surrogate host for the expression of actinobacterial Type III PKS genes: expression of the reintroduced gcs gene from S. coelicolor and of the heterologous rppA gene from Streptomyces venezuelae under the control of the constitutive ermE* promoter resulted in copious production of germicidin and flaviolin, respectively.
\end{abstract}

Conclusions: The newly constructed expression host S. coelicolor M1317 should be particularly useful for the discovery and analysis of new Type III polyketide metabolites.

Keywords: Streptomyces, Expression host, Type III polyketide synthases

\section{Background}

Type III polyketides are generally small molecules generated by Type III polyketide synthases (PKSs). These multifunctional ketosynthases typically utilise free CoA thioesters as substrates without the involvement of an acyl carrier protein (ACP), and frequently catalyse specific cyclization reactions found in aromatic and pyrone polyketides $[1,2]$. The recent explosion in genome sequencing, combined with bioinformatic analysis, has led to the identification of numerous uncharacterized Type III PKS genes in plants, fungi and bacteria, including actinomycetes $[1,3-6]$. Bacterial Type III PKSs are attractive to

\footnotetext{
*Correspondence: arinthip.t@ku.ac.th

${ }^{3}$ Center for Advanced Studies in Tropical Natural Resources, NRU-KU,

Kasetsart University, Bangkok 10900, Thailand

Full list of author information is available at the end of the article
}

study for a number of reasons: they are widely distributed, make a variety of structurally different products and, compared to Type I and Type II PKSs, relatively simple to manipulate genetically [7]. Some Type III PKSs are solely responsible for product formation. For example, germicidin synthase (Gcs) and the 1,3,6,8-tetrahydroxynaphthalene (THN) (RppA) synthases, commonly found in Streptomyces spp., are the only enzymes required to produce germicidin and THN, respectively $[5,8,9]$. THN can oxidise spontaneously to yield the red-brown pigment flaviolin, which is involved in bacterial melanin production, although a cytochrome P450 monooxygenase was reported to be involved in the conversion of THN to flaviolin in Streptomyces antibioticus $[6,10,11]$. Some Type III PKS genes are organized in small operons, such as srs $A B C$ of Streptomyces griseus that produces alkylresorcinols 
[12]. Such operons may also reside in complex gene clusters where they provide a specific precursor for the biosynthesis of more elaborate specialized metabolites; for example, the $\operatorname{dpg} A B C D$ operon that synthesizes dihydroxyphenylacetic acid which is used as a precursor for vancomycin, balhimycin, teicoplanin and kendomycin biosynthesis in Amycolatopsis orientalis [13], A. balhimycina [14], Actinoplanes teichomyceticus [15] and Streptomyces violaceoruber [16], respectively.

Within the actinomycetes, Streptomyces species such as Streptomyces lividans TK24, Streptomyces coelicolor, Streptomyces avermitilis and Streptomyces albus J1074 $[17,18]$ have been used as surrogate hosts for the heterologous expression of gene clusters encoding specialized metabolites. S. avermitilis, the industrial producer of avermectin $[19,20]$, and S. coelicolor, for many years the model species for the study of the genetics and biology of actinomycetes [21], have both been manipulated genetically to enhance the level of expression of heterologous gene clusters [22-25], and derivatives of each have been used to produce a range of different heterologous specialized metabolites [23, 24, 26-32]. In this work, we have further engineered S. coelicolor M1152 [24], a derivative of strain M145 from which four of the known antibiotic biosynthetic gene clusters had been deleted and which contains a mutation $(\mathrm{C} 1298 \mathrm{~T})$ in $r p o B$ that increases the level of secondary metabolite production, by removing all of its native Type III PKS genes. Deletion of the resident Type III PKS genes could potentially increase precursor pool levels and prevent undesirable interference with biosynthesis encoded by heterologous Type III PKS genes.

\section{Results and discussion}

\section{Type III PKS genes in S. coelicolor}

Analysis of the genome sequence of S. coelicolor M145 revealed three endogenous Type III PKS genes: sco1206, sco7221 and sco7671 [33]. sco1206 encodes RppA which synthesizes 1,3,6,8-tetrahydroxynaphthalene (THN), the intermediate involved in bacterial melanin biosynthesis, by the condensation of five malonyl CoA molecules [8]. sco1206 lies upstream of sco1207 and sco1208 (Fig. 1), which are homologues of the characterized cytochrome P450 genes mel [6] and momA [10] in S. griseus and S. antibioticus, respectively; in each of these species, the single $\mathrm{P} 450$ gene lies adjacent to a rppA homologue. $S$. coelicolor thus differs in possessing two tandemly arranged P450 genes $3^{\prime}$ of the Type III PKS gene, with all three genes apparently translationally coupled. MomA was predicted to play a role in the oxidation of THN to form flaviolin [10], while Mel was thought to couple molecules of THN to generate hexahydroxyperylenequinone melanin [6]. Since S. coelicolor is not known to produce any of these three molecules, this gene cluster may not be expressed under commonly used laboratory growth conditions. sco7221 was shown to play a role in germicidin biosynthesis [5, 34] and its product thus designated as germicidin synthase (Gcs). Analysis of Sco7671 revealed closest homology to a family of mycobacterial Type III PKSs [34]. sco7671, sco7669 and sco7670 are likely to form an operon (Fig. 1) organized in a similar fashion to srs $A B C$ of $S$. griseus (known to produce phenolic lipids, the alkylresorcinols) [12]. Although the function, substrate specificity and the product profile of $s c 07671$ have been investigated in vitro [34], the final product of the gene cluster has not been identified. To generate a clean host for the heterologous expression of Type III PKS gene clusters, we set out to delete sco7221 (gcs), sco 7669-76707671 (the srs operon) and sco1206-1207-1208 (the rppA operon) from S. coelicolor M1152 [24].

\section{Deletion of the three native Type III PKS genes and operons of S. coelicolor M1152}

All three Type III PKS genes and operons were deleted from S. coelicolor M1152 by PCR targeting [35, 36] (Fig. 2; see reference 35 for a detailed description of PCR targeting). For two of the mutations ( $\Delta$ sco7221 and $\Delta$ sco7669-7670-7671), in-frame deletions were generated in a two-step process; in the first step, the entire gene or operon in the corresponding cosmid was replaced with an apramycin (Apr) resistance cassette in Escherichia coli and the mutant allele then introduced into S. coelicolor M1152 to create an $\mathrm{Apr}^{\mathrm{R}}$-marked mutant; in the second step, the Apr ${ }^{\mathrm{R}}$ cassette in the cosmid was eliminated in E. coli using FLP-recombinase to leave an $81 \mathrm{bp}$ scar sequence which was then introduced into the $\mathrm{Apr}^{\mathrm{R}}$ marked mutant, replacing the $\mathrm{Apr}^{\mathrm{R}}$ cassette and creating an in-frame deletion mutant. Deletion of gcs (sco7221; $1122 \mathrm{bp}$ ) yielded M1314, which was then used for the in-frame deletion of the srs operon (sco7669-76707671; $2786 \mathrm{bp}$ ) to yield the double Type III PKS deletion mutant, M1316. Finally, the rppA operon (sco1206-12071208; $2859 \mathrm{bp)}$ was removed from M1316 by replacement with the $\mathrm{Apr}^{\mathrm{R}}$ cassette to yield the triple mutant M1317 (Fig. 2). Confirmation of the mutations in M1314, M1316 and M1317 was achieved by PCR amplification using primers flanking the deleted regions of the chromosome followed by DNA sequencing (data not shown). With the previous removal of four antibiotic biosynthetic gene clusters (to generate M1152), the resulting septuple mutant M1317 has lost approximately $178 \mathrm{~kb}$ of chromosomal DNA compared to the parental strain M145.

All three deletion mutants grew and sporulated in a manner that was indistinguishable from M1152 when cultures were grown on SFM agar (data not shown). 


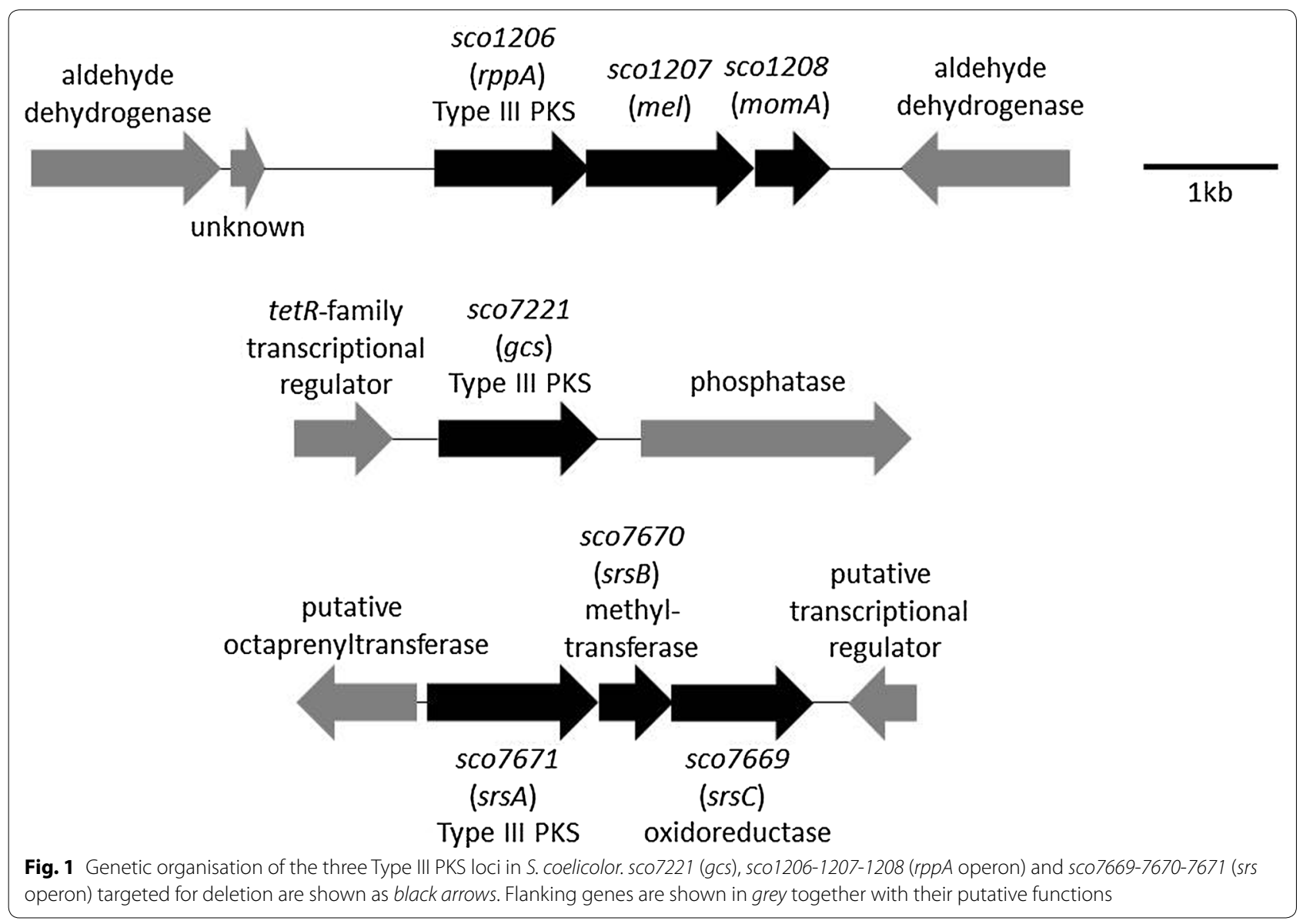

\section{Validation of S. coelicolor M1317 as a host for the expression of Type III PKS genes}

The well-characterized germicidin synthase gene of S. coelicolor was chosen initially to validate the utility of M1317 as a host for the expression of Type III PKS genes. gcs (sco7221), which is capable of producing five congeners [5], was inserted downstream of the constitutive ermE* promoter in two bifunctional multi-copy vectors, pIJ86 and pIJ12477 (a derivative of pIJ86 conferring kanamycin resistance), to give pIJ86 + sco7221 and pIJ12477 + sco7221, respectively. pIJ86 + sco7221 and pIJ86 were then transferred by conjugation into M1152 to yield M1152/pIJ86 + sco7221 and M1152/pIJ86, respectively, while pIJ12477 + sco7221 and pIJ12477 were transferred similarly to M1317 to obtain M1317/ pIJ12477 + sco7221 and M1317/pIJ12477, respectively. Germicidin production was assessed by HPLC analysis of 5 days culture supernatants of all four strains (Fig. 3a), and the masses of the peaks corresponding to each germicidin congener [5] confirmed by mass spectrometry. While no production was observed, as expected, in $\mathrm{M} 1317 / \mathrm{pI} 12477$, peaks corresponding to most of the germicidin congeners were detected in culture supernatants from the other three strains, with total production levels in M1317/pIJ12477 + sco7221 and M1152/ pIJ86 + sco7221 10.7- and 7.8-fold higher than in M1152/ pIJ86, presumably at least partly attributable to the multi-copy nature of the vectors; production in M1317/ pIJ12477 + sco7221 was 1.4-fold higher than in M1152/ pIJ86 + sco 7221, potentially reflecting increased precursor supply in the septuple deletion mutant. In each case, germicidin A and germicidin B7 were the major products (Fig. 3; Table 1).

To evaluate the use of M1317 as a host for the heterologous expression of Type III PKS genes, the rppA homologue of $S$. venezuelae (sven5367) was cloned in pIJ12477 to yield pIJ12477 + sven5367 which was introduced into M1152 and M1317 to produce M1152/ pIJ12477 + sven5367 and M1317/pIJ12477 + sven5367, respectively. Both strains produced a red-brownish pigment on IPM agar and in GYM liquid medium that was not observed in strains carrying the vectors alone (M1152/pIJ86 and M1317/pIJ12477) (Fig. 4). RppA initially generates 1,3,6,8-tetrahydroxynapthalene (THN) which is rapidly converted to flaviolin $[6,8]$. Since we were unable to detect THN by chromatography, we 


\section{S. coelicolor M1152}

(Parental strain: S. coelicolor M145 $\Delta a c t \Delta r e d \Delta c p k \Delta c d a$ rpoB [C1298T])

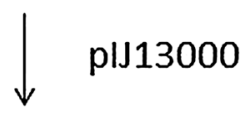

S. coelicolor M1152 $\Delta s c o 7221::(\operatorname{oriT}-a a c(3) / V)$<smiles>C=C[AsH2][Mg]</smiles>

S. coelicolor M1314

(Single mutant: M1152 $\triangle s c o 7221$ )<smiles>CC[18OH]</smiles>

S. coelicolor M1314 $\Delta$ sco7669-7670-7671::(oriT-aac(3)IV)<smiles></smiles>

S. coelicolor M1316

(Double mutant: M1314 $\Delta$ sco7669-7670-7671)

plJ13002

\section{S. coelicolor $\mathrm{M} 1317$}

(Triple mutant: M1316 $\Delta s c 01206-1207-1208::($ oriT-aac(3)IV))

Fig. 2 Construction of Type III PKS deletion mutants of S. coelicolor M1 152. Mutagenized cosmids introduced into each strain are indicated by their plJ numbers

instead quantified flaviolin production. The mass of the HPLC peak corresponding to flaviolin was confirmed by mass spectrometry [37] (Fig. 5a). Flaviolin production was readily detected in both $\mathrm{M} 1152 / \mathrm{pIJ} 12477+$ sven 5367 and M1317/pIJ12477 + sven5367, while none was detected in M1152/pIJ86 and M1317/pIJ12477 (Fig. 5b; Table 1), thus establishing the utility of M1317 as a host for the heterologous expression of Type III PKS genes. Production in M1317/pIJ12477 + sven5367 was 1.3-fold higher than in M1152/pIJ12477 + sven5367, again potentially reflecting increased precursor supply in the septuple deletion mutant. While some bacterial Type III PKSs, like plant chalcone and stilbene synthases [7], are sufficient alone for final product formation (e.g. the germicidin synthase of S. coelicolor [5] and the THN synthases of several Streptomyces species $[8,38,39])$, some require a cytochrome P450 encoded by a neighbouring gene to convert the intermediate they produce into the final product, e.g. the THN synthase of S. antibioticus [10]. Interestingly, while
rppA of $S$. venezuelae lies adjacent to a gene encoding a cytochrome $\mathrm{P} 450$ that may be required for flaviolin biosynthesis in that strain, expression of $\operatorname{rpp} A$ alone was all that was required for heterologous production of flaviolin in S. coelicolor. It is possible that in this strain THN is either oxidized spontaneously to flaviolin or oxidized by one of the remaining $18 \mathrm{P} 450$ monooxygenases encoded by the $S$. coelicolor genome [40].

Our inability to detect flaviolin production in M1152/ pIJ86 is consistent with previous work that also suggested that sco1206 is not expressed in S. coelicolor under standard laboratory conditions [8]. However, when the $\operatorname{rpp} A$ operon of S. coelicolor (sco1206-1207-1208) expressed from the $e r m E^{*}$ promoter was introduced into M1317 to yield M1317/pIJ12477 + sco1206-1207-1208, redbrown pigment production occurred in both liquid- and agar-grown cultures. Flaviolin production in M1317/ pIJ12477 + sco1206-1207-1208 was confirmed by HPLC analysis and mass spectrometry (data not shown). 

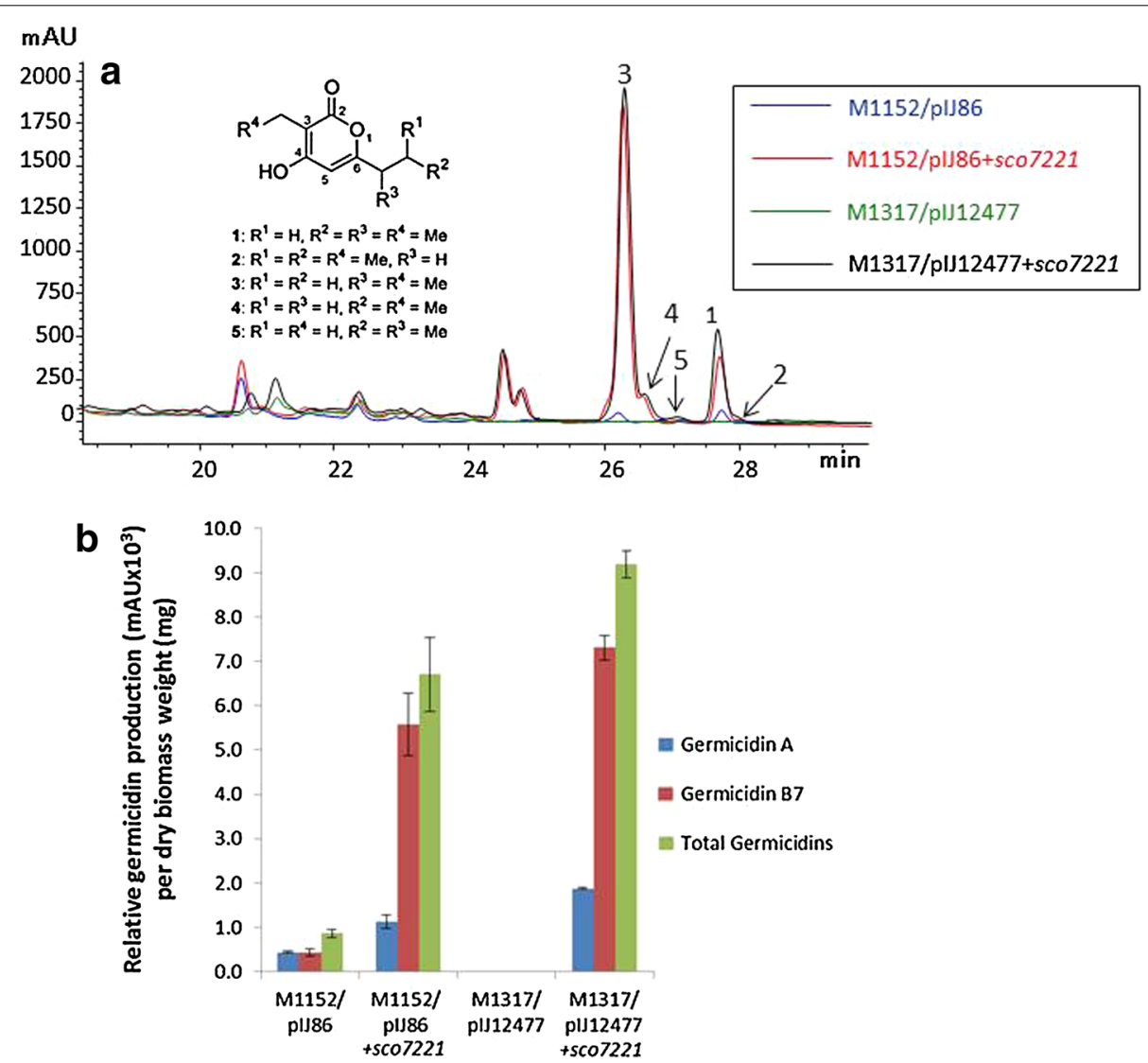

Fig. 3 Germicidin production in derivatives of S. coelicolor M1152. a HPLC chromatogram and chemical structures of germicidin derivatives [5]. Mass spectroscopy confirmed the production of the following five germicidin congeners: $1,[\mathrm{M}+\mathrm{H}]^{+}=197 \mathrm{~m} / \mathrm{z} ; 2,[\mathrm{M}+\mathrm{H}]^{+}=197 \mathrm{~m} / \mathrm{z} ; 3$, $[\mathrm{M}+\mathrm{H}]^{+}=183 \mathrm{~m} / \mathrm{z} ; 4[\mathrm{M}+\mathrm{H}]^{+}=183 \mathrm{~m} / \mathrm{z}$ and $5,[\mathrm{M}+\mathrm{H}]^{+}=183 \mathrm{~m} / \mathrm{z}$. The HPLC profiles for M1152 and M1317 were indistinguishable from those for M1152/plJ86 and M1317/pIJ12477, respectively (data not shown). b Germicidin A, germicidin B7, and total germicidin production per mg dry weight of mycelium. The histograms show the average values obtained from duplicate cultures with the two individual measurements (Table 1) indicated by bars

Table 1 Relative levels of production of germicidins and flaviolin in engineered strains

\begin{tabular}{|c|c|c|c|c|}
\hline \multirow[t]{2}{*}{ Strain } & \multicolumn{4}{|c|}{ Relative production $\left(\mathrm{mAU} \times 10^{3}\right) / \mathrm{mg}$ dry weight } \\
\hline & Germicidin A & Germicidin B7 & Total germicidins & Flaviolin \\
\hline M1152/plJ86 & $0.44 \pm 0.02$ & $0.42 \pm 0.08$ & $0.86 \pm 0.10$ & None \\
\hline M1317/pIJ12477 & None & None & None & None \\
\hline M1152/plJ86 + sco7221 & $1.13 \pm 0.14$ & $5.58 \pm 0.70$ & $6.71 \pm 0.84$ & ND \\
\hline M1317/plJ12477 + sco7221 & $1.88 \pm 0.03$ & $7.32 \pm 0.27$ & $9.19 \pm 0.30$ & ND \\
\hline M1152/plJ12477 + sven5367 & ND & ND & ND & $1.72 \pm 0.27$ \\
\hline M1317/plJ12477 + sven5367 & ND & ND & ND & $2.16 \pm 0.05$ \\
\hline
\end{tabular}

ND not determined

\section{Conclusions}

We have successfully engineered the expression host $S$. coelicolor M1152 by removing all three of the endogenous Type III PKS gene and operons, and validated the resulting septuple deletion M1317 as a host for the ectopic and heterologous expression of Type III PKS genes. We believe that S. coelicolor M1317 will prove to be a useful strain for the future analysis of Type III PKS genes and their products from a wide range of actinomycetes. 


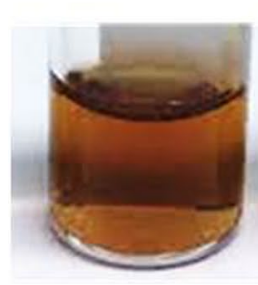

a

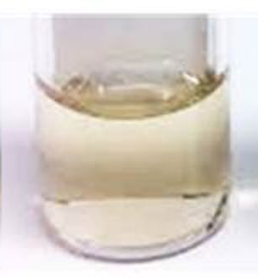

b

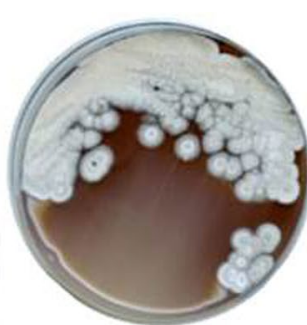

C

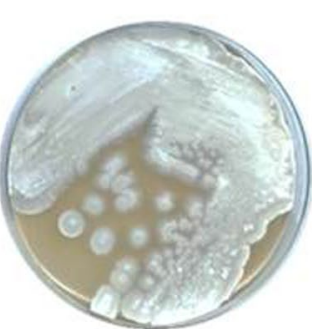

d

Fig. 4 Red-brown pigment production after heterologous expression of the rppA homologue of S. venezuelae in S. coelicolor M1317/ plJ12477 + sven5367 (a, c) compared to the same host containing the empty expression vector (M1317/pIJ12477) (b, d). a, b cultures grown in GYM liquid medium; $\mathbf{c}, \mathbf{d}$ cultures grown on IPM agar
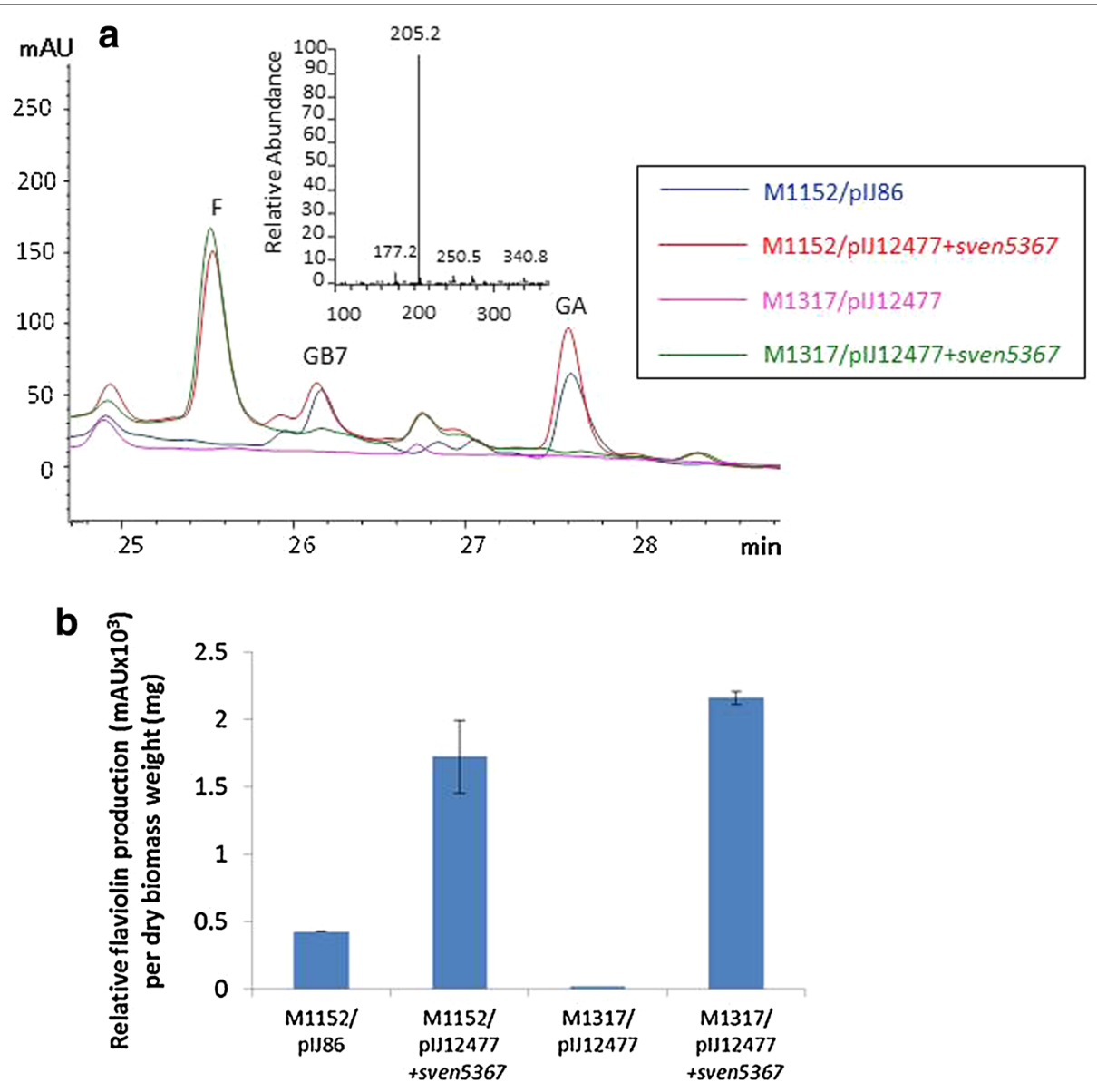

Fig. 5 Flaviolin production in derivatives of S. coelicolor M1152. a HPLC chromatogram of flaviolin (F) production in M1152/pIJ12477 + sven5367 and M1317/plJ12477 + sven5367; in each case, a mass of [M + H] $205 \mathrm{~m} / \mathrm{z}$ was confirmed by mass spectrometry (data for M1317/ pIJ12477 + sven5367 is shown in the inset). GA and GB7 indicate peaks of germicidin A and germicidin B7, respectively. The HPLC profiles for M1152 and M1317 were indistinguishable from those for M1152/plJ86 and M1317/pIJ12477, respectively (data not shown). b Flaviolin production per mg dry biomass weight. The histograms show the average values obtained from duplicate cultures with the two individual measurements (Table 1) indicated by the bars

\section{Methods}

Bacterial strains, plasmids, cosmids and culture conditions Bacterial strains, plasmids and cosmids used and generated in this study are listed in Table 2. Escherichia coli strains were grown and manipulated following standard methods [35, 36, 41]. Media for the growth of 
Table 2 Bacterial strains, plasmids and cosmids used and generated in this study

\begin{tabular}{|c|c|c|}
\hline Strain/plasmid/cosmid & Description & Source of reference \\
\hline \multicolumn{3}{|l|}{ Strain } \\
\hline E. coli BT340 & $\mathrm{DH} 5 \alpha / \mathrm{pCP} 20$ & {$[45]$} \\
\hline E. coli BW25113 & K-12 derivative: $\triangle a r a B A D, \triangle r h a B A D$ & [46] \\
\hline E. coli DH5 $\alpha$ & $\begin{array}{l}\left.\text { For cloning and propagation of host strain, deoR recA1 endA1 hsdR17(rk-, mk+ } k^{+}\right) \text {phoA supE44 thi-1 } \\
\text { gyrA96 relA1 } \lambda^{-}\end{array}$ & Invitrogen, USA \\
\hline E. coli ET12567 & dam-13::Tn9 dcm-6 hsdM hsdS & [47] \\
\hline S. coelicolor M1152 & Derivative of S. coelicolor M145, $\Delta$ act $\Delta$ red $\Delta c p k \Delta c d a r p o B(C 1298 T)$ & {$[28]$} \\
\hline S. coelicolor M1314 & S. coelicolor M1152 $\Delta$ sco 7221 & This study \\
\hline S. coelicolor M1316 & S. coelicolor M1152 $\Delta$ sco7221 $\Delta$ sco7669-7670-7671 & This study \\
\hline S. coelicolor M1317 & 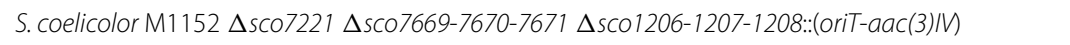 & This study \\
\hline \multicolumn{3}{|l|}{ Plasmid } \\
\hline pGEM $^{\circledR}-T$ Easy & E. coli vector for cloning PCR product, amp ${ }^{r}$ & Promega, USA \\
\hline plJ86 & ermEp*, aac(3)IV, oriT (RK2), ori (plJ101), ori (pUC18) & {$[48]$} \\
\hline plJ773 & pBluescript KS (+), aac(3)/V, oriT (RK2), FRT sites & {$[35]$} \\
\hline plJ790 & $\lambda$-RED (gam, bet, exo), cat, araC, rep $101^{\text {ts }}$ & {$[35]$} \\
\hline plJ10701 & pBluescript KS (+), hyg, oriT (RK2), bla & {$[35]$} \\
\hline plJ12477 & plJ86, neo & [49] \\
\hline plJ13000 & St2H12 $\Delta$ sco7221::(oriT-aac(3)/V) & This study \\
\hline plJ13001 & St4C2 $\Delta$ sco7669-7670-7671::(oriT-aac(3)/V) & This study \\
\hline plJ13002 & 2StG58 $\Delta$ sco1206-1207-1208::(oriT-aac(3)/M) & This study \\
\hline plJ13003 & $\mathrm{St} 2 \mathrm{H} 12 \Delta s \mathrm{co} 221$ & This study \\
\hline plJ13004 & plJ13003::(oriT-hyg) & This study \\
\hline plJ13005 & St4C2 $\Delta$ sco7669-7606-7671 & This study \\
\hline plJ13006 & plJ13005::(oriT-hyg) & This study \\
\hline plJ13007 & 2 2StG58 $\Delta$ sco 1206-1207-1208 & This study \\
\hline plJ13008 & plJ13007::(oriT-hyg) & This study \\
\hline pUZ8002 & $\mathrm{Kan}^{\mathrm{r}} / \mathrm{tra}$ neo, RP4 & {$[50]$} \\
\hline \multicolumn{3}{|l|}{ Cosmid } \\
\hline $2 S t G 58$ & SuperCos1 containing sco1206-1207-1208 & John Innes Centre, UK \\
\hline $\mathrm{St} 2 \mathrm{H} 12$ & SuperCos 1 containing sco7221 & John Innes Centre, UK \\
\hline St4C2 & SuperCos 1 containing sco $7669-7670-7671$ & John Innes Centre, UK \\
\hline Sv3G11 & SuperCos 1 containing sven 5367 & John Innes Centre, UK \\
\hline SuperCos1 & neo, bla & Agilent Technology, USA \\
\hline
\end{tabular}

S. coelicolor strains were: instant potato mash (IPM) agar [42], SFM agar [43], tryptic soy broth (TSB) and glucoseyeast extract-malt extract (GYM) medium [44]. Liquid cultures of Streptomyces strains were performed in $50 \mathrm{ml}$ of medium in $250 \mathrm{ml}$ flasks containing stainless steel springs at $30{ }^{\circ} \mathrm{C}$ with shaking at $250 \mathrm{rpm}$. The following antibiotics were used when required: $50 \mu \mathrm{g} / \mathrm{ml}$ apramycin (Apr), $50 \mu \mathrm{g} / \mathrm{ml}$ kanamycin (Kan), $50 \mu \mathrm{g} / \mathrm{ml}$ hygromycin (Hyg) and $25 \mu \mathrm{g} / \mathrm{ml}$ nalidixic acid (Nal). An overnight culture of each Streptomyces strain was used to inoculate $50 \mathrm{ml}$ of medium to give an initial $\mathrm{OD}_{600} \mathrm{~nm}=0.1$.

\section{DNA manipulations for E. coli and Streptomyces}

Extraction of DNA from $E$. coli was carried out using standard methods [36] or by using a QIAprep Spin
Miniprep Kit (QIAGEN) and following the manufacturer's instructions. Genomic DNA was isolated from Streptomyces strains using the modified Kirby Mix procedure [43]. PCR amplifications for confirmation of engineered cosmids, mutant strains and expression constructs were performed using Taq DNA polymerase (QIAGEN). Expand High Fidelity PCR System (Roche) was used to amplify disruption cassettes [35] and Phusion ${ }^{\circledR}$ HighFidelity DNA Polymerase (NEB) was used to amplify PCR products for gene expression.

\section{Deletion of the Type III PKS genes of S. coelicolor}

The three native Type III PKS genes and operons of $S$. coelicolor (sco7221, sco7669-7670-7671 and sco12061207-1208) were deleted from strain M1152 [24] by 
Table 3 Primers used in this study

\begin{tabular}{|c|c|c|}
\hline Primer & Sequence $5^{\prime}-3^{\prime}$ & Description \\
\hline plJ86F1 & ACGCCTGGTCGATGTCGGAC & \multirow{2}{*}{$\begin{array}{l}\text { Sequencing primers for recombinant plasmids of plJ86 and } \\
\text { plJ12477 }\end{array}$} \\
\hline plJ86R2 & TGCGGTCAGTGCGTGTGTCG & \\
\hline SCO1206F1 & ATCCCCAAGACCGAGGACTG & \multirow[t]{4}{*}{ Sequencing primer for internal sequence of sco 1206-1207-1208 } \\
\hline SCO1206F2 & AGGCCGTCATGGACCGCCAG & \\
\hline SCO1206F3 & TGTTCCACCTGCTGCTGAGC & \\
\hline SCO1206F4 & AGTGGTGAGCGGCCTGGTC & \\
\hline SCO1206FBgIII & AAAAAAGATCTCGCAAGCCTTCCGCGAGGCG & \multirow[t]{2}{*}{ PCR of sco1206-1207-1208 for cloning into plJ12477 } \\
\hline SCO1208RHindIII & AAAAAAAGCTTCTAACCGTGTCGCAGCGGCGTGAG & \\
\hline SCO1206FSpe & $\begin{array}{l}\text { CGCAAGCCTTCCGCGAGGCGAAAGCAGGCACGGTTCATGACTA } \\
\text { GTATTCCGGGGATCCGTCGACC }\end{array}$ & \multirow[t]{2}{*}{ Flanking primers for sco1206-1207-1208 disruption cassette } \\
\hline SCO1208RNhe & $\begin{array}{l}\text { GATCGCGCGGACGCTGGCCGGGATGCCGATCCGCTAAC- } \\
\text { CGCTAGCTGTAGGCTGGAGCTGCTTC }\end{array}$ & \\
\hline SCO1206TF & TCGAGCTGGCCAAGCTG & \multirow{6}{*}{$\begin{array}{l}\text { Test primers for verification of } \mathrm{Apr}^{\mathrm{R}} \text { cassette replacements and } \\
\text { in-frame deleted regions }\end{array}$} \\
\hline SCO1208TR & TGGAGTACGCGCAGACC & \\
\hline SCO7221TF & AGTTGCATGGGTCACTGC & \\
\hline SCO7221TR & ATGTACCGGCCCAGAGC & \\
\hline SCO7671TF & GTTGCACCAGCCGATGG & \\
\hline SCO7669TR & GTGAGTCGATGACTGTCGTGG & \\
\hline SCO7221F1 & AGTCGGTGCTCCGGCTGGAC & Sequencing primer for internal sequence of sco 7221 \\
\hline SCO7221FBamHI & AAAAAGGATCCCCTCACCTGCTCCGCAGCAGACCC & \multirow[t]{2}{*}{ PCR of sco7221 for cloning into plJ86 and plJ12477 } \\
\hline SCO7221RHindIII & AAAAAAAGCTTCTACAGCCACTCCCCTTCCAGAGCGGTGG & \\
\hline SCO7221FSpe & $\begin{array}{l}\text { CGCCGTGATACACGGCGAGCACTCCGTCGAGACCCGAGAACTA } \\
\text { GTATTCCGGGGATCCGTCGACC }\end{array}$ & \multirow[t]{2}{*}{ Flanking primers for sco7221 disruption cassette } \\
\hline SCO7221RNhe & $\begin{array}{l}\text { GTGGCGGTCAGGCCCGGGGCCGCCGCGTACAGGACG- } \\
\text { GCAGCTAGCTGTAGGCTGGAGCTGCTTC }\end{array}$ & \\
\hline SCO7671FSpe & $\begin{array}{l}\text { GTACGCACGGTAGTGGGGCCGGCGGCCGAGGAAGGCATGACTA } \\
\text { GTATTCCGGGGATCCGTCGACC }\end{array}$ & \multirow[t]{2}{*}{ Flanking primers for sco7669-7670-7671 disruption cassette } \\
\hline SCO7669RNhe & $\begin{array}{l}\text { GTCCGCTACGCGCGCGCCGCGCGGGGCCGGAACG- } \\
\text { GCTCAGCTAGCTGTAGGCTGGAGCTGCTTC }\end{array}$ & \\
\hline SVEN5367F1 & GGCCCGACACCGAGGACTG & Sequencing primer for internal sequence of sven 5367 \\
\hline SVEN5367FBamHI & AAAAAGGATCCCGGGTGAGTGTGGGCGGCAGTTC & \multirow[t]{2}{*}{ PCR of sven 5367 for cloning into pIJ12477 } \\
\hline SVEN5367RHindIII & AAAAAAAGCTTTCAGGCGACGGACGTGCGGG & \\
\hline
\end{tabular}

Bolds indicate restriction sites

PCR targeting [35, 41]. Three pairs of flanking primers, SCO7221FSpe and SCO7221RNhe, SCO7671FSpe and SCO7669RNhe, and SCO1206FSpe and SCO1208RNhe (Table 3), were designed to amplify a $1458 \mathrm{bp}$ fragment carrying an apramycin resistance $\left(\mathrm{Apr}^{\mathrm{R}}\right)$ oriT cassette from pIJ773 [35]. Each of the Apr ${ }^{\mathrm{R}}$ cassettes were flanked by $39 \mathrm{nt}$ sequences matching the ends of the gene or operon to be deleted, and the targeted regions replaced with the $\mathrm{Apr}^{\mathrm{R}}$ cassette by homologous recombination between these 39 nt sequences. Cosmids St2H12, St4C2 and 2StG58, containing sco7221, sco7669-7670-7671 and sco1206-1207-1208, respectively, were transferred into $E$. coli BW25113 [47]/pIJ790 [35] and the resulting strains were then transformed with the corresponding replacement cassette. The mutagenized cosmids pIJ13000, pIJ13001 and pIJ13002, respectively, were verified by restriction enzyme digestion and PCR amplification with primers SCO7221TF and SCO7221TR, SCO7671TF and SCO7669TR, and SCO1206TF and SCO1208TR, respectively (Table 3). pIJ13000 ( $\Delta$ sco7221::(oriT-aac(3)IV)) was then introduced into S. coelicolor M1152 by conjugation with selection for apramycin resistance; subsequent screening for loss of kanamycin resistance (encoded by the SuperCos 1 vector) but retention of apramycin resistance was used to isolate the required $\Delta s c o 7221::$ (oriT$a a c(3) I V)$ replacement mutant. In the meantime, the $\mathrm{Apr}^{\mathrm{R}}$ cassettes were removed from pIJ13000, pIJ13001 and pIJ13002 in E. coli BT340 [46] using the FLP-recombinase, in each case leaving a $81 \mathrm{bp}$ scar sequence in place of the cassette. The resulting non-transmissible cosmids, pIJ13003, pIJ13005 and pIJ13007, respectively, were confirmed by restriction enzyme digestion and PCR 
amplification using the same primer sets that were used for verification of the $\mathrm{Apr}^{\mathrm{R}}$ cassettes (Table 3). To allow transfer of these cosmids from E. coli to Streptomyces via conjugation, a bla-oriT-hyg-bla cassette was amplified from pIJ10701 [35] and used to target the bla gene in the SuperCos1 backbone of pIJ13003, pIJ3005 and pIJ13007, yielding pIJ13004, pIJ13006 and pIJ13008, respectively. Intergeneric conjugation between the $S$. coelicolor $\Delta s c o 7221::($ oriT-aac(3)IV) replacement mutant and $E$. coli ET12567/pUZ8002 [47, 50] containing pIJ13004 was performed as previously described [35, 41]. Exconjugants were selected based on hygromycin resistance and the desired $\Delta s c o 7221$ in-frame deletion mutant M1314 obtained by screening for Apr, Kan and Hyg sensitive segregants and confirmed by sequencing of the PCR product obtained from genomic DNA using primers SCO7221TF and SCO7221TR. Essentially the same procedure was used to generate the double in-frame deletion mutant M1316 ( $\Delta$ sco 7221, $\Delta$ sco7669-7671) from M1314 using pII13001 and pI)13006 (and confirmed by sequencing the PCR amplicon obtained from genomic DNA using PCR primers SCO7671TF and SCO7669TR). Similarly the triple deletion mutant M1317 ( $\Delta$ sco7221, $\Delta$ sco7669-7671, $\Delta s c 01206-1208:: a a c(3) I V$ ) was derived from M1316 using $\mathrm{pI} 13002$. Despite repeated attempts, it was not possible to remove the $\mathrm{Apr}^{\mathrm{R}}$ cassette from M1317 using pIJ13008. Therefore, M1317 was confirmed by sequencing of the PCR product obtained from genomic DNA using PCR primers SCO1206TF and SCO1208TR. In each case the PCR fragment was cloned into pGEM ${ }^{\circledR}-\mathrm{T}$ Easy and sequenced using M13 universal primers to verify that the expected deletions had been made.

The three mutants were grown on SFM agar until sporulation. Spores were harvested and stored in $20 \%$ glycerol at $-20^{\circ} \mathrm{C}$.

\section{Construction of the type III PKS expression strains}

sco7221 (gcs) and sco1206-sco1207-sco1208 (rppA operon) from S. coelicolor and sven5367 (rppA homologue) from $S$. venezuelae were amplified from cosmids St2H12, 2StG58 and Sv3G11, respectively, using primers SCO7221FBamHI and SCO7221RHindIII, SCO1206FBglII and SCO1206RHindIII, and SVEN5367FBamHI and SVEN5367RHindIII, respectively (Table 3). The forward (F) primers were designed to include the ribosome binding sites of sco7221, sco1206 and sven 5367, respectively. The PCR products were cloned into $\mathrm{pGEM}^{\circledR}{ }_{-}-\mathrm{T}$ Easy and confirmed by restriction enzyme digestion and DNA sequencing using M13 universal primers and the internal primers SCO7221F1 for pGEM-T/sco7221, SCO1206F1, SCO1206F2, SCO1206F3 and SCO1206F4 for pGEM$\mathrm{T} /$ sco1206-sco1207-sco1208, and SVEN5367F1 for pGEM-T/sven5367 (Table 3). Two multi-copy expression vectors, each containing the constitutive $e r m E^{*}$ promoter, were used; pIJ86 [48] was employed in M1152, while pIJ12477 [49] (a derivative of pIJ86 also conferring kanamycin resistance) was used in M1152 and in M1317. sco7221 was sub-cloned as a BamHI-HindIII fragment into both pIJ86 and pIJ12477, yielding pIJ86 + sco 2221 and pIJ12477 + sco7221, respectively. sco1206-sco1207sco1208 (rppA operon) were sub-cloned as a BglII-HindIII fragment into BamHI plus HindIII cleaved pIJ12477, yielding pIJ12477 + sco1206-sco1207-sco1208. sven 5367 was sub-cloned as a BamHI-HindIII fragment into pIJ12477 to obtain pIJ12477 + sven5367. Primers pIJ86F1 and pIJ86R2 (Table 3), which anneal 220 and 229 bp from the BamHI and HindIII cloning sites, respectively, were used to verify the recombinant plasmids by PCR amplification and sequencing in addition to restriction enzyme digestion. For pIJ12477 + sco1206-sco1207-sco1208, primers pIJ86F1 and the internal primers SCO1206F1, SCO1206F2, SCO1206F3 and SCO1206F4 were also used for sequencing. The plasmids were then introduced into E. coli ET12567/pUZ8002 by transformation and transferred to the appropriate Streptomyces host by conjugation as described previously [43]. Exconjugants were selected on SFM agar containing $50 \mu \mathrm{g} / \mathrm{ml}$ apramycin or $50 \mu \mathrm{g} / \mathrm{ml}$ kanamycin as appropriate and $25 \mu \mathrm{g} / \mathrm{ml}$ nalidixic acid. Spore stocks were prepared on SFM agar containing the appropriate antibiotic for plasmid selection and stored in $20 \%$ glycerol at $-20{ }^{\circ} \mathrm{C}$.

\section{Analysis of germicidin and flaviolin production}

Cultures were grown for 5 days in $50 \mathrm{ml}$ of GYM liquid medium, $5 \mathrm{ml}$ of each culture supernatant lyophilized, the dried samples dissolved in $500 \mu \mathrm{l}$ water and filtered through a VectaSpin Micro Polysulphone $0.2 \mu \mathrm{m}$ column (Whatman). The samples were injected onto a Phenomenex Gemini-NX3u C18 110A, $150 \times 4.6$ mm column fitted to an Agilent 1100 HPLC system and analyzed using a linear methanol:water gradient in $0.1 \%(\mathrm{w} / \mathrm{v})$ formic acid as follows: 0 min, $10 \%$ methanol; $1 \mathrm{~min}, 25 \%$ methanol; $21 \mathrm{~min}, 100 \%$ methanol; $25.50 \mathrm{~min}, 10 \%$ methanol; $33 \mathrm{~min}, 10 \%$ methanol, at a flow rate of $0.8 \mathrm{ml} / \mathrm{min}$ with UV absorbance monitoring at $280 \mathrm{~nm}$. Fractions of interest were collected and analyzed by mass spectrometry (Thermo Scientific ${ }^{\mathrm{TM}}$ ). Germicidins were analyzed using positive ion mode mass spectrometry whereas flaviolin was analyzed using negative ion mode. Each experiment was carried out in duplicate. The mycelium from each $50 \mathrm{ml}$ culture was harvested by centrifugation, dried and the dry weight determined. Relative levels of germicidin and flaviolin production were calculated by multiplying the average peak area (mAU) obtained from a $5 \mathrm{ml}$ sample by ten and dividing by the average dry weight obtained from a $50 \mathrm{ml}$ culture. 


\section{Abbreviations}

ACP: acyl carrier protein; Apr: apramycin; Gcs: germicidin synthase: Hyg: hygromycin; Kan: kanamycin; PKS: polyketide synthase; THN: 1,3,6,8-tetrahydroxynaphthalene.

\section{Authors' contributions}

AT, JC, JPG-E, MJB and AT conceived and designed the project. AT and JPG-E performed experiments and analyzed data. AT, MJB and AT wrote the manuscript. All authors read and approved the final manuscript.

\section{Author details}

${ }_{1}^{1}$ Department of Genetics, Faculty of Science, Kasetsart University, Bangkok 10900, Thailand. ${ }^{2}$ Department of Molecular Microbiology, John Innes Centre, Norwich NR4 7UH, UK. ${ }^{3}$ Center for Advanced Studies in Tropical Natural Resources, NRU-KU, Kasetsart University, Bangkok 10900, Thailand. ${ }^{4}$ Present Address: Department of Bioengineering and Therapeutic Sciences and the California Institute for Quantitative Biosciences, University of California San Francisco, San Francisco, CA 94158, USA.

\section{Acknowledgements}

A Thanapipatsiri has been awarded Ph.D. scholarship by the Royal Golden Jubilee of the Thailand Research Fund (RGJ-TRF). This work was supported financially by the Prime Minister's Initiative for International Education through a Connect-Research Co-operation Award administered by the British Council, UK; Kasetsart University Research and Development Institute (KURDI); and by the Biotechnological and Biological Sciences Research Council (BBSRC, UK) Institute Strategic Programme Grant "Understanding and Exploiting Plant and Microbial Secondary Metabolism" (BB/J004561/1).

\section{Compliance with ethical guidelines}

\section{Competing interests}

The authors declare that they have no competing interests.

Received: 14 June 2015 Accepted: 3 September 2015 Published online: 16 September 2015

\section{References}

1. Moore BS, Hopke JN. Discovery of a new bacterial polyketide biosynthetic pathway. Chembiochem. 2001;2:35-8.

2. Hertweck C. The biosynthetic logic of polyketide diversity. Angew Chem Int Ed Engl. 2009;48:4688-716.

3. Pfeifer V, Nicholson GJ, Ries J, Recktenwald J, Schefer AB, Shawky RM, et al. A polyketide synthase in glycopeptide biosynthesis: the biosynthesis of the non-proteinogenic amino acid (S)-3,5-dihydroxyphenylglycine. J Biol Chem. 2001;276:38370-7.

4. Saxena P, Yadav G, Mohanty D, Gokhale RS. A new family of Type III polyketide synthases in Mycobacterium tuberculosis. J Biol Chem. 2003;278:44780-90

5. Song L, Barona-Gomez F, Corre C, Xiang L, Udwary DW, Austin MB, et al. Type III polyketide synthase beta-ketoacyl-ACP starter unit and ethylmalonyl-CoA extender unit selectivity discovered by Streptomyces coelicolor genome mining. J Am Chem Soc. 2006;128:14754-5.

6. Funa N, Funabashi M, Ohnishi Y, Horinouchi S. Biosynthesis of hexahydroxyperylenequinone melanin via oxidative aryl coupling by cytochrome P-450 in Streptomyces griseus. J Bacteriol. 2005;187:8149-55.

7. Yu D, Xu F, Zeng J, Zhan J. Type III polyketide synthases in natural product biosynthesis. IUBMB Life. 2012;64:285-95.

8. Izumikawa M, Shipley PR, Hopke JN, O'Hare T, Xiang L, Noel JP, et al. Expression and characterization of the Type III polyketide synthase 1,3,6,8-tetrahydroxynaphthalene synthase from Streptomyces coelicolor A3(2). J Ind Microbiol Biotechnol. 2003:30:510-5.

9. Austin MB, Izumikawa M, Bowman ME, Udwary DW, Ferrer JL, Moore $B$, et al. Crystal structure of a bacterial Type III polyketide synthase and enzymatic control of reactive polyketide intermediates. J Biol Chem. 2004;279:45162-74.

10. Funa N, Funabashi M, Yoshimura E, Horinouchi S. A novel quinoneforming monooxygenase family involved in modification of aromatic polyketides. J Biol Chem. 2005;280:14514-23.
11. Zhao B, Guengerich FP, Bellamine A, Lamb DC, Izumikawa M, Lei L, et al. Binding of two flaviolin substrate molecules, oxidative coupling, and crystal structure of Streptomyces coelicolor A3(2) cytochrome P450 158A2. J Biol Chem. 2005;280:11599-607.

12. Funabashi M, Funa N, Horinouchi S. Phenolic lipids synthesized by Type III polyketide synthase confer penicillin resistance on Streptomyces griseus. J Biol Chem. 2008;283:13983-91.

13. Chen H, Tseng CC, Hubbard BK, Walsh CT. Glycopeptide antibiotic biosynthesis: enzymatic assembly of the dedicated amino acid monomer (S)-3,5-dihydroxyphenylglycine. Proc Natl Acad Sci USA. 2001;98:14901-6.

14. Pelzer S, Sussmuth R, Heckmann D, Recktenwald J, Huber P, Jung G, et al. Identification and analysis of the balhimycin biosynthetic gene cluster and its use for manipulating glycopeptide biosynthesis in Amycolatopsis mediterranei DSM5908. Antimicrob Agents Chemother. 1999;43:1565-73.

15. Horbal L, Kobylyanskyy A, Truman AW, Zaburranyi N, Ostash B, Luzhetskyy $A$, et al. The pathway-specific regulatory genes, tei1 $5^{*}$ and tei $16^{*}$, are the master switches of teicoplanin production in Actinoplanes teichomyceticus. Appl Microbiol Biotechnol. 2014;98:9295-309.

16. Wenzel SC, Bode HB, Kochems I, Muller R. A Type I/Type III polyketide synthase hybrid biosynthetic pathway for the structurally unique ansa compound kendomycin. Chembiochem. 2008;9:2711-21.

17. Olano C, Carmaen M, José AS. Streptomyces: Molecular Biology and Biotechnology. Gene clusters for bioactive natural products in actinomycetes and their use in combinatorial biosynthesis. Norfolk: Caister Academic Press; 2011.

18. Baltz RH. Streptomyces and Saccharopolyspora hosts for heterologous expression of secondary metabolite gene clusters. J Ind Microbiol Biotechnol. 2010;37:759-72.

19. Omura S, Ikeda H, Ishikawa J, Hanamoto A, Takahashi C, Shinose M, et al. Genome sequence of an industrial microorganism Streptomyces avermitilis: deducing the ability of producing secondary metabolites. Proc Natl Acad Sci USA. 2001;98:12215-20.

20. Ikeda H, Ishikawa J, Hanamoto A, Shinose M, Kikuchi H, Shiba T, et al. Complete genome sequence and comparative analysis of the industrial microorganism Streptomyces avermitilis. Nat Biotechnol. 2003;21:526-31.

21. Bentley SD, Chater KF, Cerdeno-Tarraga AM, Challis GL, Thomson NR, James KD, et al. Complete genome sequence of the model actinomycete Streptomyces coelicolor A3(2). Nature. 2002;417:141-7.

22. Komatsu M, Uchiyama T, Omura S, Cane DE, Ikeda H. Genome-minimized Streptomyces host for the heterologous expression of secondary metabolism. Proc Natl Acad Sci USA. 2010;107:2646-51.

23. Komatsu M, Komatsu K, Koiwai H, Yamada Y, Kozone I, Izumikawa M, et al. Engineered Streptomyces avermitilis host for heterologous expression of biosynthetic gene cluster for secondary metabolites. ACS Synth Biol. 2013;2:384-96.

24. Gomez-Escribano JP, Bibb MJ. Engineering Streptomyces coelicolor for heterologous expression of secondary metabolite gene clusters. Microb Biotechnol. 2011;4:207-15.

25. Gomez-Escribano JP, Bibb MJ. Streptomyces coelicolor as an expression host for heterologous gene clusters. Methods Enzymol. 2012;517:279-300.

26. Li T, Du Y, Cui Q, Zhang J, Zhu W, Hong K, et al. Cloning, characterization and heterologous expression of the indolocarbazole biosynthetic gene cluster from marine-derived Streptomyces sanyensis FMA. Mar Drugs. 2013;11:466-88.

27. Niu G, Li L, Wei J, Tan H. Cloning, heterologous expression, and characterization of the gene cluster required for gougerotin biosynthesis. Chem Biol. 2013;20:34-44.

28. Flinspach K, Westrich L, Kaysser L, Siebenberg S, Gomez-Escribano JP, Bibb M, et al. Heterologous expression of the biosynthetic gene clusters of coumermycin $A(1)$, clorobiocin and caprazamycins in genetically modified Streptomyces coelicolor strains. Biopolymers. 2010;93:823-32.

29. Flinspach K, Kapitzke C, Tocchetti A, Sosio M, Apel AK. Heterologous expression of the thiopeptide antibiotic GE2270 from Planobispora rosea ATCC 53733 in Streptomyces coelicolor requires deletion of ribosomal genes from the expression construct. PLoS One. 2014. doi:10.1371/journal.pone.0090499.

30. Robles-Reglero V, Santamarta I, Alvarez-Alvarez R, Martin JF, Liras P. Transcriptional analysis and proteomics of the holomycin gene cluster in overproducer mutants of Streptomyces clavuligerus. J Biotechnol. 2013;163:69-76. 
31. Jankowitsch F, Schwarz J, Ruckert C, Gust B, Szczepanowski R, Blom J, et al. Genome sequence of the bacterium Streptomyces davawensis JCM 4913 and heterologous production of the unique antibiotic roseoflavin. J Bacteriol. 2012;194:6818-27.

32. Ikeda H, Kazuo SY, Omura S. Genome mining of the Streptomyces avermitilis genome and development of genome-minimized hosts for heterologous expression of biosynthetic gene clusters. J Ind Microbiol Biotechnol. 2014;41:233-50.

33. Challis GL, Hopwood DA. Synergy and contingency as driving forces for the evolution of multiple secondary metabolite production by Streptomyces species. Proc Natl Acad Sci USA. 2003;100(Suppl 2):14555-61.

34. Gruschow S, Buchholz TJ, Seufert W, Dordick JS, Sherman DH. Substrate profile analysis and ACP-mediated acyl transfer in Streptomyces coelicolor Type III polyketide synthases. Chembiochem. 2007;8:863-8.

35. Gust B, Chandra G, Jakimowicz D, Yuqing T, Bruton CJ, Chater KF. Lambda red-mediated genetic manipulation of antibiotic-producing Streptomyces. Adv Appl Microbiol. 2004;54:107-28.

36. Sambrook J, Russell DW. Molecular cloning: a laboratory manual. New York: Cold Spring Harbor Laboratory Press; 2001.

37. Beltran-Garcia MJ, Prado FM, Oliveira MS, Ortiz-Mendoza D, Scalfo AC, Pessoa A Jr, et al. Singlet molecular oxygen generation by light-activated DHN-melanin of the fungal pathogen Mycosphaerella fijiensis in black Sigatoka disease of bananas. PLoS One. 2014. doi:10.1371/journal. pone.0091616.

38. Ghimire GP, Oh TJ, Liou K, Sohng JK. Identification of a cryptic Type III polyketide synthase (1,3,6,8-tetrahydroxynaphthalene synthase) from Streptomyces peucetius ATCC 27952. Mol Cells. 2008;26:362-7.

39. Funa N, Ohnishi Y, Ebizuka Y, Horinouchi S. Properties and substrate specificity of RppA, a chalcone synthase-related polyketide synthase in Streptomyces griseus. J Biol Chem. 2002;277:4628-35.

40. Lim YR, Hong MK, Kim JK, Doan TT, Kim DH, Yun CH, et al. Crystal structure of cytochrome P450 CYP105N1 from Streptomyces coelicolor, an oxidase in the coelibactin siderophore biosynthetic pathway. Arch Biochem Biophys. 2012;528:111-7.

41. Gust B, Challis GL, Fowler K, Kieser T, Chater KF. PCR-targeted Streptomyces gene replacement identifies a protein domain needed for biosynthesis of the sesquiterpene soil odor geosmin. Proc Natl Acad Sci USA. 2003;100:1541-6.

42. Joshi MV, Mann SG, Antelmann H, Widdick DA, Fyans JK, Chandra G, et al The twin arginine protein transport pathway exports multiple virulence proteins in the plant pathogen Streptomyces scabies. Mol Microbiol. 2010;77:252-71.

43. Kieser T, Bibb MJ, Buttner MJ, Chater KF, Hopwood DA. Practical Streptomyces Genetics. Norwich Research Park, Colney, Norwich: John Innes Foundation; 2000.

44. Shima J, Hesketh A, Okamoto S, Kawamoto S, Ochi K. Induction of actinorhodin production by rpsL (encoding ribosomal protein S12) mutations that confer streptomycin resistance in Streptomyces lividans and Streptomyces coelicolor A3(2). J Bacteriol. 1996;178:7276-84.

45. Cherepanov PP, Wackernagel W. Gene disruption in Escherichia coli: TcR and KmR cassettes with the option of Flp-catalyzed excision of the antibiotic-resistance determinant. Gene. 1995;158:9-14.

46. Datsenko KA, Wanner BL. One-step inactivation of chromosomal genes in Escherichia coli K-12 using PCR products. Proc Natl Acad Sci USA. 2000;97:6640-5.

47. MacNeil DJ, Gewain KM, Ruby CL, Dezeny G, Gibbons PH, MacNeil T. Analysis of Streptomyces avermitilis genes required for avermectin biosynthesis utilizing a novel integration vector. Gene. 1992;111:61-8.

48. Healy FG, Eaton KP, Limsirichai P, Aldrich JF, Plowman AK, King RR. Characterization of gamma-butyrolactone autoregulatory signaling gene homologs in the angucyclinone polyketide WS5995B producer Streptomyces acidiscabies. J Bacteriol. 2009;191:4786-97.

49. Claesen J. Cloning and analysis of the cypemycin biosynthetic gene cluster. University of East Anglia; 2010.

50. Paget MS, Chamberlin L, Atrih A, Foster SJ, Buttner MJ. Evidence that the extracytoplasmic function sigma factor sigmaE is required for normal cell wall structure in Streptomyces coelicolor A3(2). J Bacteriol. 1999;181:204-11.

\section{Submit your next manuscript to BioMed Central and take full advantage of:}

- Convenient online submission

- Thorough peer review

- No space constraints or color figure charges

- Immediate publication on acceptance

- Inclusion in PubMed, CAS, Scopus and Google Scholar

- Research which is freely available for redistribution

Submit your manuscript at

www.biomedcentral.com/submit

C BioMed Central 